\title{
HUBBLE SPACE TELESCOPE OBSERVATIONS OF AGN*
}

\author{
F. MACCHETTO** \\ Space Telescope Science Institute
}

\section{Introduction}

Only little more than three years have passed since the launch of the Hubble Space Telescope (HST) and the wealth of results produced by astronomers using it, have already made fundamental contributions to our understanding of a variety of astrophysical processes. A considerable number of investigations have been, and are being, devoted to the study of the whole gamut of problems associated with activity in galaxies. These range from the very largest scales, namely those applicable to the study of the optical jets and galaxy mergers $(10-100 \mathrm{kpc})$ to the smallest scales $(1-10 \mathrm{pc})$ relevant to investigate the broad-line regions and the very center of the active galaxies. In all cases, the high-spatial resolutions, extended dynamic range and ultraviolet response, has made possible the study of a number of objects with a detail impossible without the HST.

In a broad sense, the results so far have generally confirmed, the "standard" unified picture of AGN, providing new observational constraints to the model; however, a number of notable exceptions or "complications" have been unveiled which will require considerable more theoretical work and even more precise observations with the refurbished HST to resolve.

In this review, I have followed the following scheme. The first two sections discuss the large-scale phenomena, jets and mergers, which are a manifestation of, or the cause of the AGN activity. In the subsequent sections I discuss some of the most recent and important results in the three subclasses of active galaxies proper and follow the "standard paradigm" as its backdrop, highlighting where appropriate the observations that do not conform to our current picture. In all cases, I have used only results of articles recently appeared in, or submitted to, the main journals or conference proceedings.

\section{Optical Jets}

The study of the optical counterparts to the radio jets has been the subject of a number of observing programs. We know that these jets play a fundamental role in transporting energy from the central source to the extended radio lobes. Observations at optical and ultraviolet wavelengths with the HST are essential to obtain spatial resolutions similar to, or better than, those achieved in the radio band

* Based on observations obtained with the NASA/ESA Hubble Space Telescope

** On assignment from the Space Science Department of ESA

T. J.-L. Courvoisier and A. Blecha: Multi-Wavelength Continuum Emission of AGN, 83-104.

(c) 1994 IAU. Printed in the Netherlands. 
and, thus, provide the possibility of directly comparing the sites and mechanisms responsible for the emission at these different wavelengths.

In all cases to date, the emission has been attributed to the synchrotron mechanism, and since the electron lifetime is a strong function of the observed frequency, observations at optical and ultraviolet wavelengths offer the possibility to determine the precise locations where particle acceleration occurs. Comparison of the radio and optical morphologies further allows the study of the confinement mechanisms and diffusion processes within the jet.

A number of important discoveries and observations that place the theoretical models on firmer observational grounds have been published or are about to be published: PKS 0521-36 (Macchetto et al 1991a), 3C66B (Macchetto et al 1991b), NGC 3862 (Crane et al 1993), M87 (Macchetto, 1991a,b; Boksenberg et al. 1992; Lauer et al. 1992) and have been reviewed in the past (Macchetto 1991a,b; 1992; 1993.) Particularly, interesting results have been published recently on $3 \mathrm{C} 273$ and M87.

3C273 is one of the nearest and brightest quasars known. Its jet has been extensively studied at radio and optical wavelength, the latter, however, limited by the low resolution attainable from the ground. Using the Faint Object Camera on HST, Thomson, Mackay and Wright (1993) have carried out high-resolution imaging polarimetric observations of the jet. Fig. 1 shows a ground-based image of 3C273 and its jet and the total intensity image of the jet as observed with the HST. The projected jet length is more than $70 \mathrm{kpc}\left(H_{0}=50 \mathrm{~km} \mathrm{~s}^{-1} \mathrm{Mpc}^{-1}\right.$; $\left.q_{0}=0.5\right)$. The width is only a few tenths of an arcsecond $(\sim 0.5-1 \mathrm{kpc})$. The optical emission is highly confined to the core of the radio jet. It runs along the ridge of the radio emission and is asymmetric compared to the radio.

The bright optical photo shows polarization contours in excess of $20 \%$ and the magnetic and polarization vectors are generally aligned along the jet near the quasar, but the orientation changes along the jet and they seem to twist by $40^{\circ}$ and become parallel with the inclined inner structure of the outer knot. The HST data are consistent with pure synchrotron radiation as the source of both optical and radio emission, and is contrary to the suggestion that the optical emission is simply quasar light scattered in our direction by jet particles.

The authors interpret these results as a continuous fluid model in which a jet of ionized plasma burrows its way through the intergalactic medium, and where the outermost knot, and its rotated magnetic field angle, is the working surface of the jet. The asymmetrical optical and radio morphologies are interpreted as a transverse motion of the jet, due to either a processing jet or a transverse wind.

The authors also discuss the possibility that the jet is intrinsically one-sided, but confirmation of this model requires considerable more observational and theoretical work.

Optical and ultraviolet observations of $\mathbf{M 8 7}$ have been carried out with HST and have been extensively reported (Macchetto 1991a,b, 1992, 1993; Boksenberg et al. 1992; Lauer et al. 1992; Macchetto, Biretta and Sparks 1993). The early 

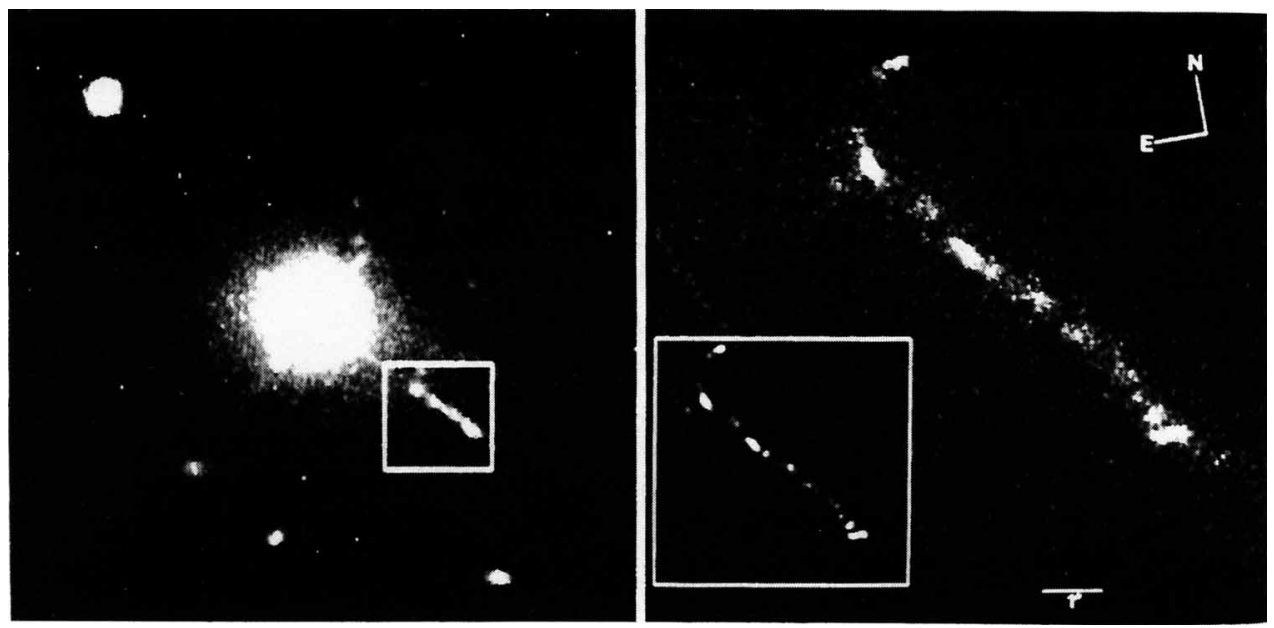

Fig. 1 Ground-based (left) and HST images of the 3 C273 jet.

HST /FOC observations have demonstrated a remarkable similarity between the radio and optical morphologies. However, more detailed comparisons (Sparks, Biretta \& Macchetto 1993) highlight the several systematic differences between these different wavelength bands.

The optical emission appears to be more concentrated in the knots than the radio emission. The ratio of knot to interknot brightness is higher for the optical data. This is evident in Fig. 2 where the radio image shows a diffuse component of emission which fills the inner jet (knots D, E, F, and I), while any such component is much weaker in the optical images. This is also apparent in the spectral index map, Fig. 3 where the centers of the knots have indices $\alpha \leq 0.6$ whereas the interknot regions are appreciably steeper with $\alpha \geq 0.7$. To confirm this effect, we have also compared the VLA and HST images with ground-based NTT, $V$ and $R$ band data. In both the HST and NTT data, the ratio of knot to interknot brightness is higher than in the radio.

The jet also appears narrower in the optical than in the radio band. This is most easily seen at knot A where the FWHM normal to the jet axis is about $1.13^{\prime \prime}$ in the VLA data, but only $0.85^{\prime \prime}$ in the HST FOC data. All of the knots show the 
same effect to varying degrees.

Besides these systematic trends, there are also localized regions which are relatively much brighter in the HST data than in the radio. This is most apparent in knots $E$ and $F$ of the inner jet.

The jet also appears narrower in the optical than in the radio band. This is most easily seen at knot A where the FWHM normal to the jet axis is about $1.13^{\prime \prime}$ in the VLA data, but only $0.85^{\prime \prime}$ in the HST FOC data. All of the knots show the same effect to varying degrees.

Besides these systematic trends, there are also localized regions which are relatively much brighter in the HST data than in the radio. This is most apparent in knots $E$ and $F$ of the inner jet.

There are two spectral effects which might account for the systematic variations in the radio to optical spectral index found here. A sharp spectral break where the spectrum steepens by $\Delta \alpha \sim 0.6$ is known to occur between the infrared and optical bands. Fluctuations in the frequency of this break would produce small changes in the radio-to-optical spectral index, even though all regions might have the same spectral shape. The observed spectral variations would indicate the magnetic fields are stronger at the jet center, and inside the knots, than elsewhere. While such a structure is possible, it is contrary to recent suggestions of magnetic confinement and evidence for a low-loss channel at the jet center.

An alternative possibility is that the interknot and jet-edge spectra are steeper throughout the entire electromagnetic spectrum. This picture gains some support from preliminary measurements of the interknot radio spectral index. Here the particle energy spectra would be steeper in these regions, perhaps due to variations in the acceleration process.

Future observations of the interknot radio spectral index, as well as deeper HST images giving the interknot optical spectra, will help decide between these pictures.

\section{Mergers}

As part of the standard paradigm, galaxy-mergers have become accepted as a key ingredient in either producing an AGN or in triggering activity in a "dormant" nucleus (e.g., Heckman 1992, and references therein). Numerical simulations have shown (Barnes \& Haernquist 1991) that tidal interactions or mergers of galaxies can be responsible for supplying large amounts of gas and dust to the innermost regions of a galaxy and, thus, be responsible for resupplying the central engine (black hole?) with the necessary fuel to account for the observed activity. While direct evidence that AGN's are fueled via mergers or other accretion processes is not (yet!) overwhelming, the amount of indirect evidence continues to increase, and HST observations at high resolution are contributing to better define this picture.

Mrk 315 is a moderately luminous Seyfert 1.5 galaxy and has a redshift of $11,820 \mathrm{~km} \mathrm{~s}^{-1} ; M_{V}=-21.6$. The scale is $0.57 h^{-1} \mathrm{kpc} \operatorname{arcsec}^{-1}$. It is a steep spectrum source with a diffuse morphology and a total extent of $2.9 h^{-1} \mathrm{kpc}$. This 


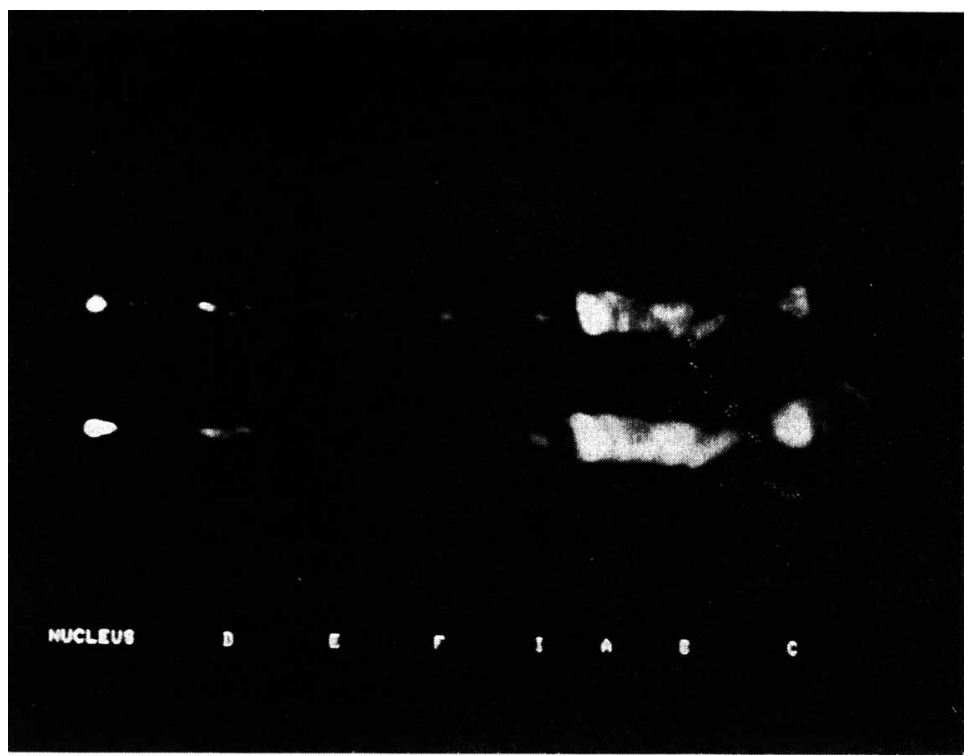

Fig. 2 The M87 jet as seen by the FOC (top) and the VLA (bottom). Note that the knot to interknot brightness ratio is higher in the optical data.

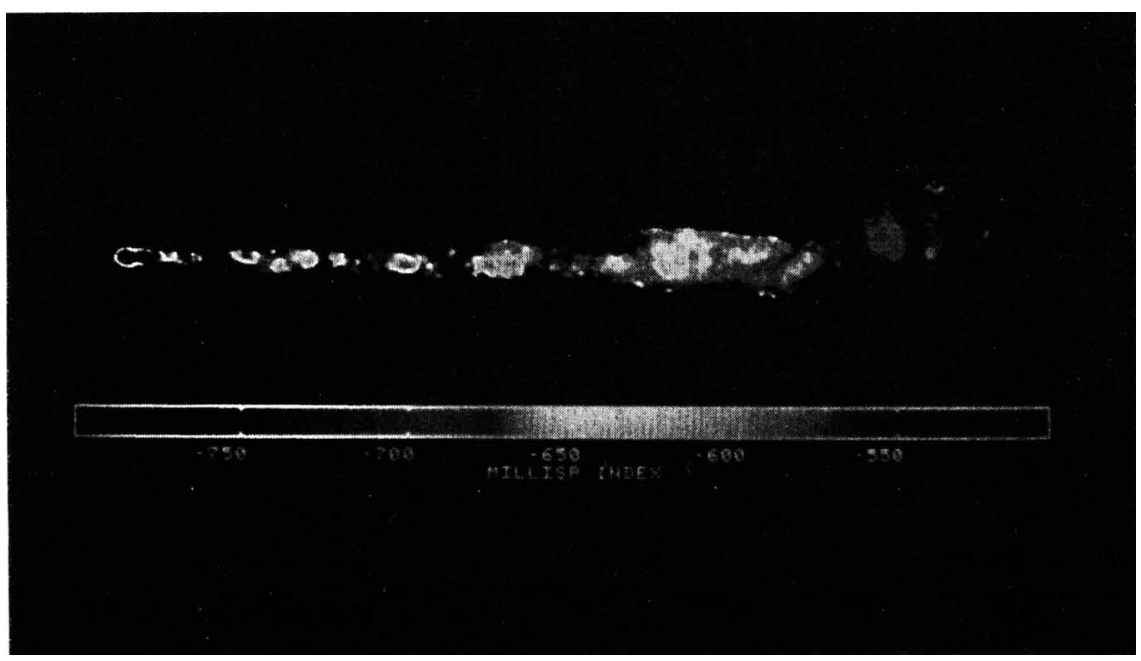

Fig. 3 Radio-to-optical spectral index map for the M87 jet. 
structure is consistent with an extended starburst in the galaxy and the IRAS fluxes are also consistent with this interpretation. Mackenty (1986) discovered an $80 \mathrm{kpc}$ streamer of ionized gas emerging from near the nucleus, extending in a straight line for $60 \mathrm{kpc}$ then bending back in a hook. He suggested two possible origins for this feature: a tidal interaction or a dormant radio jet.

The HST observations, Fig. 4, (Mackenty et al. 1993) support the idea of a tidal origin and establish Mrk 315 as an extreme example of an active galaxy with a strong tidal disruption in the gas-rich material close to its nucleus.

The HST image shows a second, diffuse peak 2.27 " east of the diffraction limited, stellar Seyfert nucleus. This second peak is clearly resolved with a Gaussian FWHM of $0.66^{\prime \prime}$ (corresponding to $380 \mathrm{~h}^{-1} \mathrm{pc}$ at the distance of Mrk 315). A fainter ring, or spiral-like structure is present $2.5^{\prime \prime}-3^{\prime \prime}$ south of the nucleus $(\sim 1.4 \mathrm{kpc})$ opening towards the SW. The brighter of the two nuclei is the core of Mrk 315 and probably contains a massive black hole. The fainter nucleus is considered to be the surviving core of a galaxy that recently merged into Mrk 315 .

The features seen in the data for Mrk 315 are consistent with an interpretation of the secondary continuum knot being a recently captured galaxy remnant. The knot is at the leading arc of what appears to be a stellar, spiral density enhancement. The inner ionized gas structures and the VLA $20 \mathrm{~cm}$ morphology are consistent with recent star formation, and subsequent supernova explosions in gas compressed by tidally induced cloud-cloud collisions. The asymmetrical velocity pattern in the gas kinematics suggests that the center of gravity for the system is displaced from the Seyfert nucleus. All of these different features are symptomatic of the type of gravitational forces which leads to mass inflow towards the nucleus.

NGC 7252 is the prototypical example of a merger between two disk galaxies. It shows two long tidal tails, one of which extends to $\sim 280^{\prime \prime}(130 \mathrm{kpc})$ from the center of the galaxy. The outer regions show clear evidence of a recent interaction involving two disks, while the inner region of NGC 7252 appears to have relaxed into a relatively spheroidal distribution. Schweizer (1982); found that the luminosity profile follows a $r^{1 / 4}$ law, and discovered a counterrotating disk of ionized gas within $8^{\prime \prime}$ of the center, with a $1^{\prime \prime}-2^{\prime \prime}$ hole at its center.

Whitmore et al. (1993), report on observations made with the Planetary Camera of the HST, Fig. 5. They discovered a population of about 40 blue pointlike objects in this galaxy with a mean absolute magnitude of $M_{V}=-13$ mag; mean color $V-I=0.7 \mathrm{mag}$; and mean effective radius of $10 \mathrm{pc}$ (for $H_{0}=50 \mathrm{~km} \mathrm{~s}^{-1} \mathrm{Mpc}^{-1}$ ). The luminosities, colors, projected spatial distribution, and sizes are all compatible with the hypothesis that these object formed within the last $1 \mathrm{Gyr}$ following the collision of two spiral galaxies, and that they are young globular clusters. They conclude that new globular clusters can be created during a merger between two gas-rich galaxies.

Whitmore et al. also discovered a bright spiral structure within $3.5^{\prime \prime}(1.6 \mathrm{kpc})$ of the center, with dust lanes and weak spiral structure extending out to about $9^{\prime \prime}(4.2 \mathrm{kpc})$. This structure closely corresponds to the counterrotating disk of 


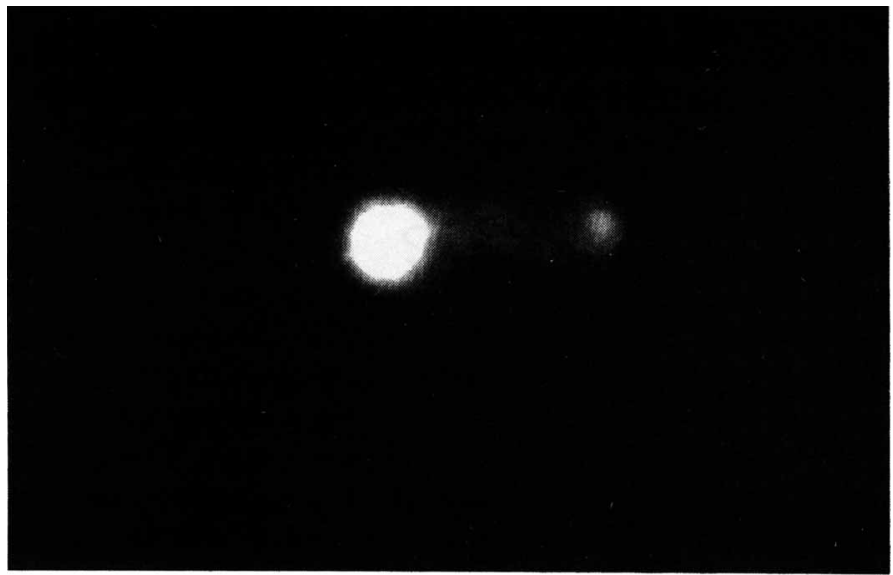

Fig. 4 HST view of the nucleus of Mrk 315. The diffuse peak is 2.3" east of the stellar Seyfert nucleus.

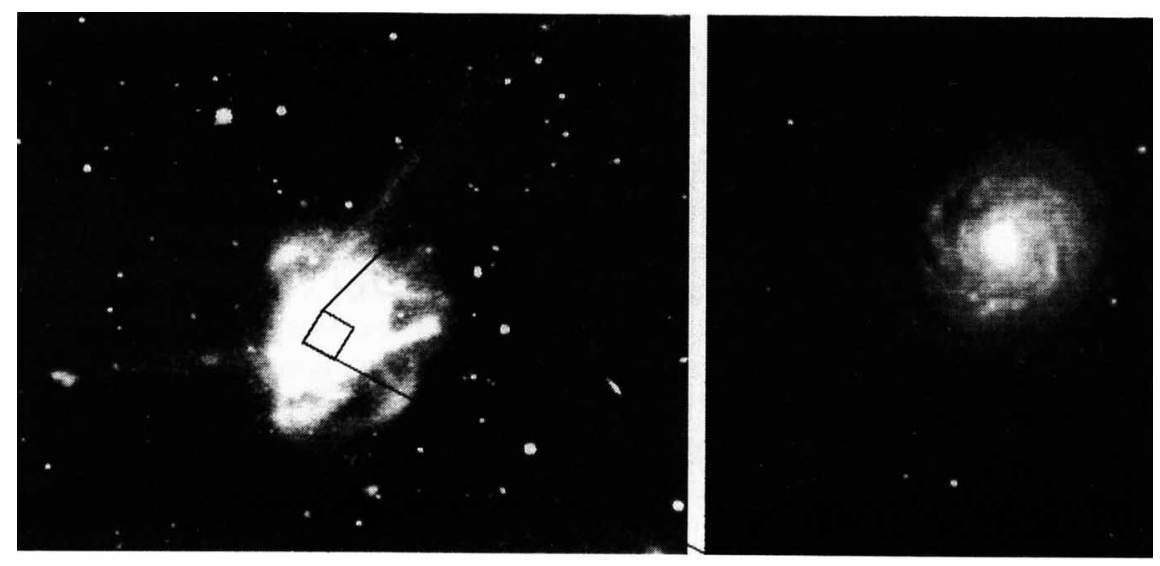

Fig. 5 Ground-based (left) and HST (right) images of NGC 7252. The inner spiral structure has a diameter of only 9 " $(4.2 \mathrm{kpc})$. 
ionized gas observed by Schweizer (1982), and is presumably formed through the continued infall of gas into this central disk. This infall would be sufficient to trigger the activity of an AGN, if one existed in this galaxy.

Arp 220 is a low surface brightness galaxy with a well defined dust lane along its center. IRAS observations have shown that it emits $1.2 \times 10^{12} L_{\odot}$, at far infrared wavelengths, and thus, it is the prototype of ultraluminous IR galaxies, with a farinfrared luminosity that exceeds its optical luminosity by one order of magnitude. The best candidate mechanisms for this enormous amount of energy are either an AGN, or a large population of bright young stars. In both cases, the trigger is thought to be a galaxy merger in the recent past.

Planetary Camera images of Arp 220 were taken in the $V, R$, and $I$-band filters, Fig. 6 by Shaya et al. (1993). They show a very luminous object near the position of the western radio continuum source and 7 fainter objects within $2^{\prime \prime}$ of this position. The most luminous object appears to coincide with the radio source, however, an alternate alignment of maps shows the eastern radio source to coincide with one of the fainter objects and the $\mathrm{OH}$ radio sources to coincide with yet other objects. The authors favor the interpretation of those objects as massive young star associations with luminosities $10^{9}-10^{11} L_{\odot}$, but highly extinguished by intervening dust. These massive associations should fall into the nucleus on a timescale of $10^{8}$ years. This implies that the associations are young and that dynamical friction is a very efficient mechanism to funnel large amounts of material into the obscured nucleus.

Assuming that all of these objects are young associations, between $10 \%-20 \%$ of the far-IR flux could arise from these objects depending on how many remain totally obscured. In addition, if the diffuse starlight out to a radius of $8^{\prime \prime}$ is dominated by stars with typical ages of order $10^{8}$ years, the time since the merger of two galaxies, then the reradiation of diffuse starlight contributes at least $3 \times 10^{11} L_{\odot}$ to the far-infrared flux, or $\geq 25 \%$ of the total far-IR flux.

The additional bright objects $\left(M_{V} \approx-13\right)$ located about $5^{\prime \prime}$ from the core are likely protoglobular clusters, but any of these could be recently exploded supernovae instead. The expected supernova rate, if the dominant energy source is young stars, is about one per month for the region where the anomalous far-infrared flux originates. Also, individual giant dust clouds are visible in these images. Their typical size is $300 \mathrm{pc}\left(1^{\prime \prime}\right)$.

\section{Active Galaxies}

In the standard and, perhaps, simplistic unified model of AGN, the basic differences between the various classes of active galaxies can be explained as the result of varying orientations to the line of sight, (see Antonucci, 1993 for a review).

All active galaxies have a central energy source (e.g., black-hole) surrounded by the broad-line region and optical featurless continua. In turn, these are surrounded by an optically thick torus, which is oriented perpendicular to the radio jets (in 


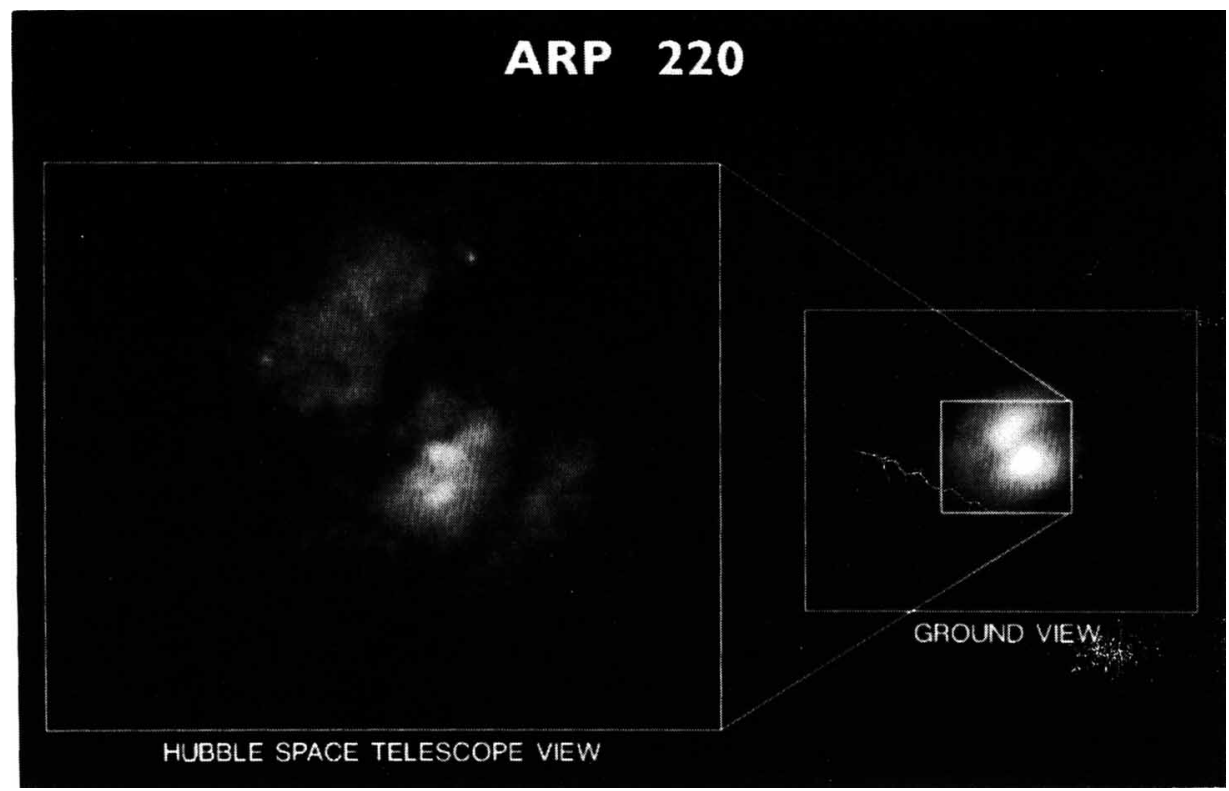

Fig. 6 HST (left) and ground-based (right) views of Arp 220. Note the resolved dust lane and the bright regions.

the case of radio-loud galaxies). All sources have, therefore, the same intrinsic geometry, although a wide range of luminosities is possible.

Those galaxies seen face-on are defined spectroscopically as Type 1 and have wide permitted lines in emission from the broad-line region, as well as narrowemission lines, both permitted and forbidden from the narrow-line region. Type 1 galaxies also show strong featurless continua which are almost always observed to be variable.

Type 2 galaxies do not show emission from the broad-line region, nor a strong nuclear featurless continuum. HST observations of the different types of AGN seem to conform to this broad picture. However, in all cases, they considerably expand our understanding and in some cases (e.g., NGC 4151), they challenge the standard scenario.

\subsection{TyPE 1 GalaXies}

A number of Seyfert 1 galaxies have been studied with HST, e.g., M51 (Ford et al 1993); NGC 4395 (Fillipenko et al 1993), etc. Of particular interest are recent 
observations of two radio-loud galaxies NGC 4261 and 3C449.

The radio source $3 \mathrm{C} 270$ has been mapped at various frequencies, and consists of a nuclear point source and two jets with outer lobes.

Observations of NGC 4261 (3C270) with the HST have been reported by Jaffe et al (1993), Fig. 7.

Jaffe et al, discovered a large $2 \times 10^{20} \mathrm{~cm}$ radius disk of cool dust and gas surrounding the bright unresolved nucleus of NGC 4261. They suggest that the bright point corresponds to thermal emission from the hot disk and that this is fueled by material flowing from the cool 'outer' accretion disk. The spin axis of the accretion disk is parallel to the direction of the radio jets.

The emission in the outer areas of the HST picture arises from normal early type stars; their density increases smoothly towards the nucleus. The centre of the image shows a well-defined disk that absorbs the light from the stars behind it. At the centre of the disk is the bright unresolved nucleus. The disk itself is elliptical, with axes of $1.71^{\prime \prime} \times 0.74^{\prime \prime}$, and its major axis is at a position angle of $-16^{\circ}$.

For an intrinsically circular disk, this implies that the plane of the disk is inclined $64^{\circ}$ to the plane of the sky. Since the distance of NGC 4261 is $14.7 \mathrm{Mpc}\left(1^{\prime \prime}=71\right.$ pc), the projected disk size is $121 \mathrm{pc} \times 51 \mathrm{pc}$.

The position angle of the disk's major axis coincides with that of the galaxy as a whole but, it is worth noting that the galaxy rotates about its major axis, rather than its minor axis.

The absorption in the disk is presumably caused by interstellar dust embedded in cool gas. The jet axis is parallel to the spin axis of the disk, and thus, is perpendicular to the rotation axis of the galaxy. This strongly suggests that the angular momentum of the disk is responsible for the orientation of the jets. The effect might be caused either by transfer of angular momentum from the disk to a central black hole, or by formation of a jet 'nozzle' in the inner disk by hydrodynamic forces. The disk observed in the HST picture is $10^{5}-10^{7}$ times larger than the presumed inner accretion disk. Jaffe et al, estimate that the timescale for the viscous transport of 'fuel' into the central engine is about $10^{8} \mathrm{yr}$., which is compatible with the timescale and energy requirements for the radio jet.

HST observations of the low luminosity radio-source $3 \mathrm{C449}$ have been discussed by Capetti, Macchetto, Sparks \& Miley (1994). This source has two extended $\left(>15^{\prime}\right)$ radio jets, which display a remarkable mirror symmetry. Ground-based observations failed to detect any optical counterpart to the radio jets (Fraix-Burnet et al. 1991). Because of its relatively close distance $\left(z=0.017 ; 0.1^{\prime \prime}=50 h_{50}^{-1} \mathrm{pc}\right)$, this CD galaxy is ideal for high resolution studies with the HST. The observations were obtained with the Faint Object Camera, and show a 23 mag. nucleus surrounded by a ring whose projected radius is $0.4^{\prime \prime}$, Fig. 8 . The ring center is coincident with the nucleus and has an ellipticity of $0.11 \pm 0.09$ and a position angle of its major axis of $\theta=81 \pm 10$ degrees. Assuming cylindrical symmetry for the inner region, this corresponds to an angle between the axis of the ring and the line of sight of $\phi=27 \pm 12$ degrees. The de-projected radius is $0.20^{\prime \prime}<r<0.23^{\prime \prime}$ 


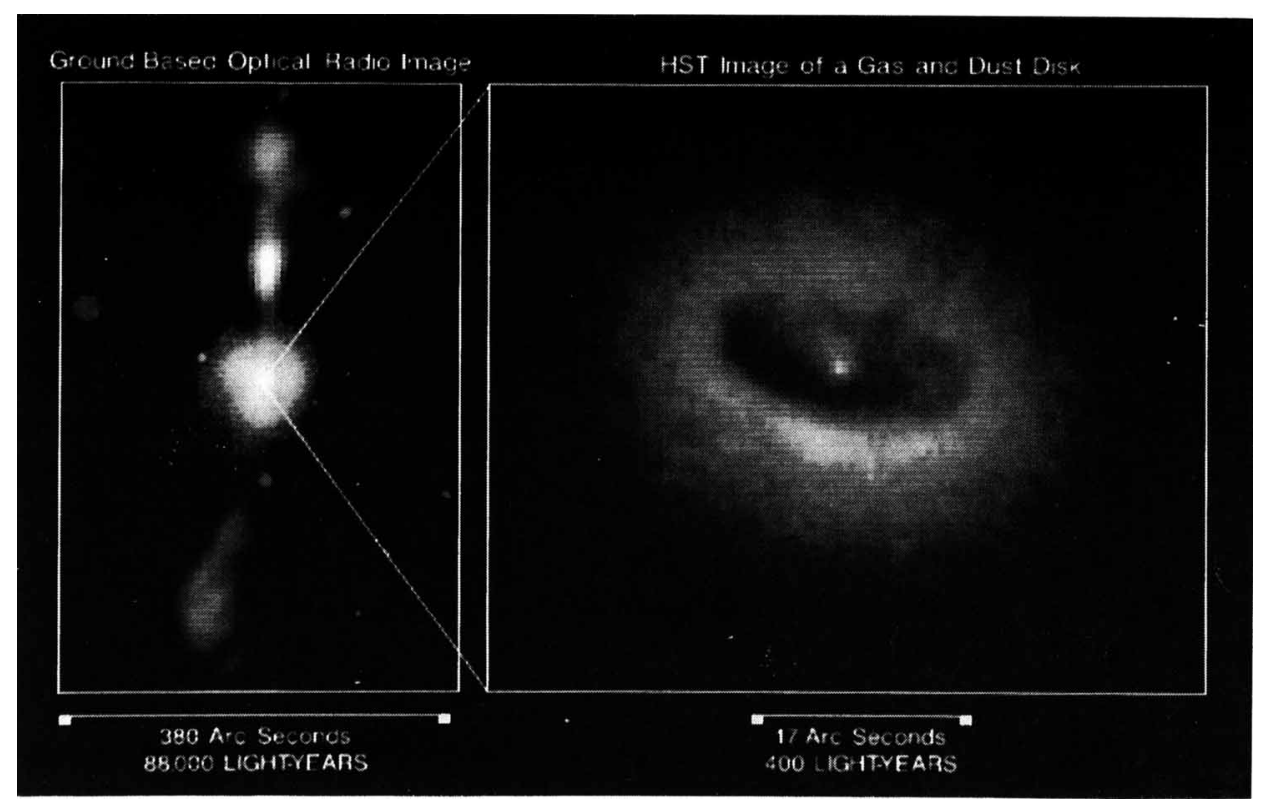

Fig. 7 Composite optical and radio image of NGC 4261 (left) and the HST image (right). The extended accretion disc is $1.7^{\prime \prime} \times 0.7^{\prime \prime}$

corresponding to about $100 \mathrm{pc}$. The luminosity profile is well fitted by an $r^{1 / 4}$ law for $r>0.3^{\prime \prime}$, about the radius of the ring, but in the inner region, the $r^{1 / 4}$ law overestimates the observed light profile.

The interpretation is that the nuclear region is strongly absorbed and the observed ring is actually produced by the high brightness gradient and the contrast with the inner absorbed region. The size of this region is consistent with it being due to cool material associated with an extended-accretion disk which partially absorbs the light of the underlying galaxy. This is also supported by the close alignment of the ring axis to the radio axis and by the presence of two bright and compact emitting regions found North and South of the nucleus.

The two bright knots are observed at a distance from the nucleus of about $0.55^{\prime \prime}$. The angle between the knots and the core is 167 degrees, about the same angle formed by the radio jets. There is an angular displacement of $15^{\circ}$ between the jets and the line joining the optical knots and the nucleus, but this difference can be explained with lateral oscillations of the radio-jets in the inner 10", oscillations that are required by the observed radio morphology at larger distance. Therefore, it is 
very likely that the radio-jets are responsible for the formation of the optical knots. Most, if not all, of the flux from the knots appears to be continuum emission. This essentially rules out the possibility that the ionization is produced by the nuclear source, supporting the interpretation that the radio-jets are producing the knots.

Therefore, we conclude that the natural explanation for the peculiar morphology observed in 3C449 is that we are seeing material associated with an extended accretion disk obscuring the inner region of the galaxy. The jets originating from the nucleus at the center of the accretion disk produce two optical knots, probably associated with shock waves internal to the jets.

\subsection{Transition Type Galaxies}

When mother nature wants to remind us that our attempts at describing the physical phenomena with "standard paradigms" or "grand unified theories" are not to be taken too seriously, it provides us with objects such as NGC 4151 that "almost" fit our models. In this case, we call them "transition" objects (or Seyferts 1.5 !), since they display characteristics of more than one class. We study these type of objects in great detail, because we can learn a great deal about the fundamental physics by how well they do or do not fit in detail our models.

NGC 4151 is the nearest ( $30 \mathrm{Mpc}$ ) example of a (sometime) type 1 Seyfert galaxy. The broad emission component of $\mathrm{H} \beta$, which is characteristic of Seyfert 1 galaxies, varies dramatically and in a low state can almost disappear. This led to NGC 4151 being reclassified as Seyfert 1.5. Both the permitted and the continuum emission show variations on time scales as short as days.

HST observations of NGC 4151 at several wavelengths in the optical and ultraviolet were obtained by the FOC (Boksenberg 1992, Boksenberg et al. 1993). These include observations obtained in the line and continuum around [O III] $\lambda 5007 \AA$. The UV observations including those around C IV and Ly $\alpha$ shows strong emission arising from the nucleus. However, there is no ultraviolet counterpart to the well-defined biconical structure abong PA 245 seen in [O III]. This consists of the bright nucleus and a number of distinct emission-line clouds with typical sizes of $10 \mathrm{pc}$, which lie within the NRL. The clouds are distributed within the biconical structure with a cone opening angle of $\sim 75^{\circ} \pm 10^{\circ}$ and with apices coincident with the central point source Fig. 9. The cone is aligned with the extension of the nuclear VLB1 radio-source indicating that the same mechanism is responsible for the alignment of the optical ionizing radiation field and the parsec scale radio structure.

Long-slit spectroscopic observations carried-out with the FOC (Boksenberg et al. 1993a) show narrow-line emission components due to the bright knots within the cones and significant continuum and broad-line components, which arise from the intense nuclear emission. The radial velocity structure indicates an outflow of material along the jets, as well as material which shares in the general rotation of the galaxy; from these measurements we derive an upper limit of $4 \times 10^{8} M_{\odot}$ for the total material contained within a radius of $30 \mathrm{pc}$ of the nucleus. From a 


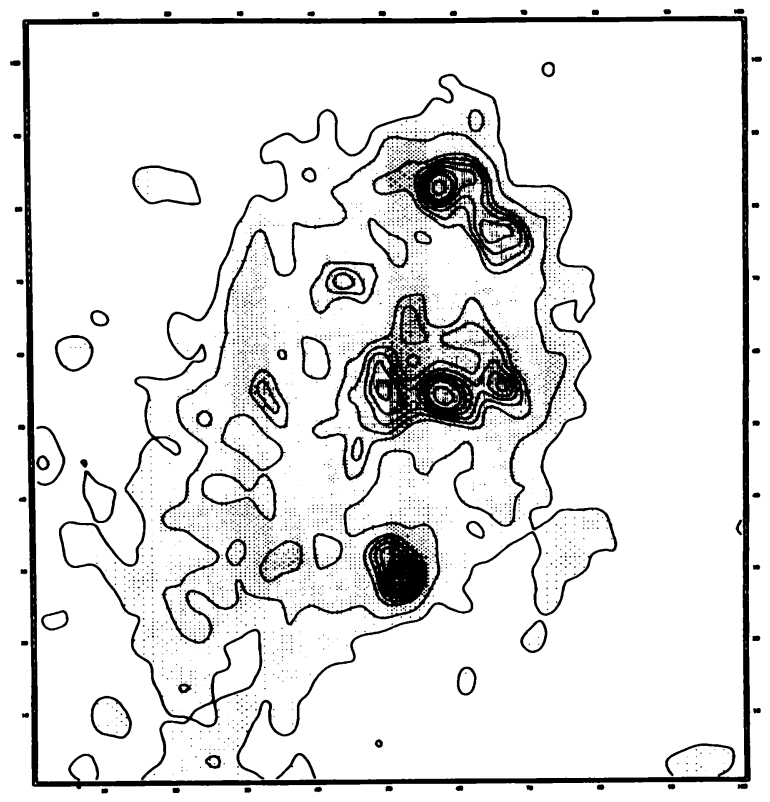

Fig. 8 In $3 \mathrm{C} 449$, the HST shows an extended accretion disc (0.2" or $100 \mathrm{pc}$ radius) and two bright knots aligned with the radio-axis.

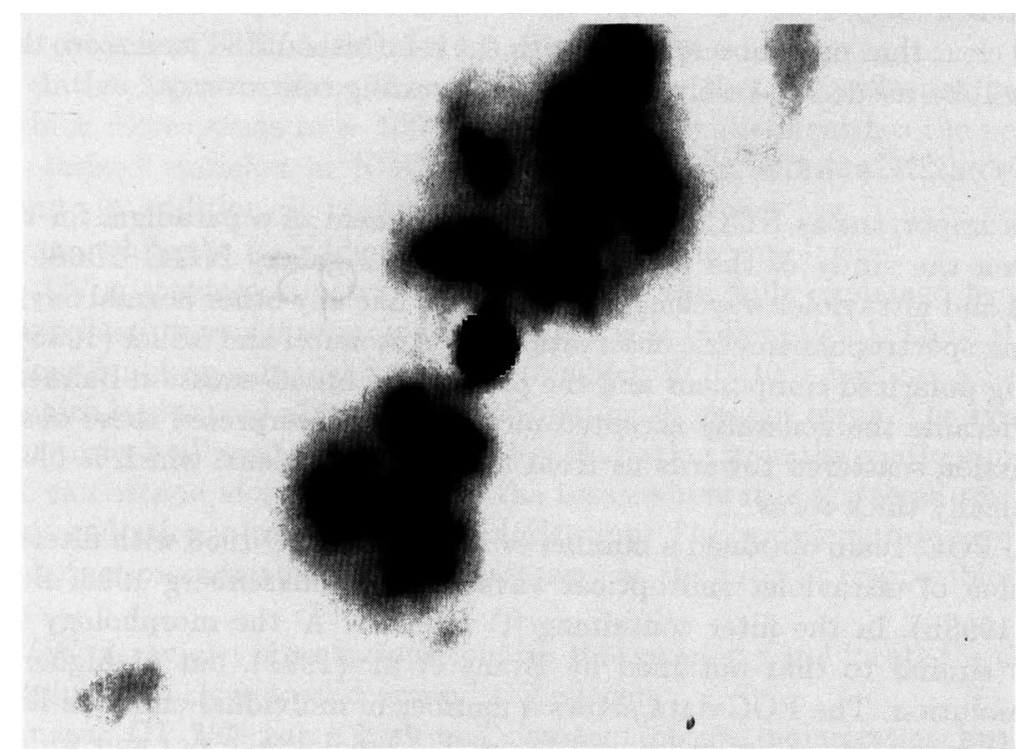

Fig. 9 This deconvolved HST image of NGC 4151 was taken in [O III] and shows a bright-nucleus and a well defined biconical structure. Typical cloud sizes are $10 \mathrm{pc}$. 
detailed comparison of the optical and radio morphology and from the ultraviolet observations, Boksenberg et al, derive a rotation of the cone position axis as a function of the distance from the nucleus and conclude that the line of sight falls within, although barely, the ionization cone.

Different conclusions have been reached by Evans et al. (1993), which using similar data obtained with the PC observe the same emission-line morphology (although without any accompanying ultraviolet data). They propose that the geometry inferred from the data is incompatible with the simplest version of the unified model of AGN, namely, a very optically thick molecular torus surrounding the BLR to obscure the source of ionizing photons.

They conclude that for feasible model parameters, the line of sight must lie outside of the ionization cone, and since the very optically thick molecular torus model would predict that the central broad-line point source should not be visible directly, the model is not correct, or does not apply to NGC 4151 .

On the other hand, obscuration models with effective neutral hygrogen column density along the line of sight of $10^{20}<N_{H}<10^{21} \mathrm{~cm}^{-2}$ are compatible with the observations.

Possible ways out include different mechanisms for collimating the radiation close to the central source: a very optically thick torus surrounded by a lower density atmosphere that collimates the ionizing radiation, but leaves the line of sight to the nucleus relatively unobscured; a disk geometry for the BLR clouds so that their opacity absorbs most of the ionizing radiation emitted in the disk plane; and finally, an optically thick, but geometrically thin accretion disk which surrounds a black-hole.

It is clear that more observations with the refurbished HST and more theoretical work will be needed to resolve this very interesting controversy.

\subsection{Type 2 Galaxies}

Just as important as NGC 4151 to the development of a paradigm for the AGN, has been the study of the prototypical Seyfert 2 galaxy NGC 1068. While at optical and ultraviolet wavelengths, it appears like any other normal Seyfert 2, pioneering spectropolarimetric observations by Antonucci and Miller (1985) revealed a strong polarized component and the presence of broad-emission Balmer lines. In what became the generally accepted picture, they interpreted these observations as emission scattered towards us from a Seyfert 1 nucleus, which is obscured by an optically thick torus.

The FOC Team obtained a number of images of NGC 1068 with filters covering a number of ultraviolet and optical wavelengths. (Boksenberg 1992, Boksenberg et al. 1993b). In the filter containing [O III] $5007 \AA$ the morphology Fig. 10 is clearly similar to that obtained by Evans et al. (1991), but at higher contrast and resolution. The FOC data shows a number of individual emission-line clouds, resolved with sizes of the order of $0.1^{\prime \prime}-0.2^{\prime \prime}\left(\sim 6-12 h_{50}^{-1} \mathrm{pc}\right)$ and which outline a clear biconical structure, with emission projecting also from South-West of the 
nucleus. The cone apex is coincident with the apex of the radio-emission suggesting that both collisional and excitation mechanisms are at play.

The ultraviolet images show high-contrast extended features clearly related to the [O III] structure, but considerably different in detail. They each show a dense diffuse concentration of emission near the nucleus which is of similar general appearance to the resolved bright feature in the optical continuum image, but which is not present in the [O III] 'pure' line image. This feature in the strong continuum component corresponding to the optical continuum emission and the result of scattering into our line of sight by the electron cloud. These observations considerably strengthen the case for the obscured-nucleus model.

Of considerable relevance are recent multiaperture spectropolarimetric observations caried-out with the FOS in the ultraviolet at wavelengths between $1575 \AA$ and $3300 \AA$ (Hurt, Antonucci \& Miller, 1993), which allowed them to investigate the scattered, nuclear light without the complication of polarization dilution caused by strong unpolarized starlight.

The main results can be summarized as follows: the UV continuum polarization is constant $\left(\mathrm{p} \sim 16 \%\right.$ at $\left.\theta \sim 97^{\circ}\right)$ from $1600 \AA$ to $2800 \AA$. The value and wavelength independence of the polarization agree with other UV observations (Code et al 1993) and with expectations from optical spectropolarimetry (Miller \& Antonucci 1983). The polarization decreases redward of $2800 \AA$ reaching a value of $\sim 11.5 \%$ at $3300 \AA$. This can be explained by dilution from unpolarized starlight and implies a starlight fraction of $\sim 30 \%$ at $3300 \AA$. The narrow lines are much less polarized than the continuum, with typical polarizations of $<2 \%$. In polarized flux, the narrow lines are absent, and two lines, C III $\lambda 1909$ and $\mathrm{Mg}$ II $\lambda 2798$ have large equivalent widths of $\sim 25 \AA$ and $\sim 14 \AA$ respectively.

The relative fluxes in these three apertures show that the reflecting region is $\sim 1.0^{\prime \prime}$ which corresponds to $\sim 100$ pc. Fig. 11 clearly distinguishes the polarized and unpolarized emission in NGC 1068. The starlight dilution at the red end is apparent. In addition, it is clear that both [C III] and $\mathrm{Mg}$ II have polarized components, while the forbidden line [Ne IV] $\lambda 2423$ is unpolarized.

These UV spectropolarimetric observations are also fully explained by the occultation/reflection model proposed by Antonucci \& Miller (1985). Thus, the HST observations build up a model according to which NGC 1068 harbors a Seyfert 1 nucleus which is obscured along the line of sight by an opaque torus. The symmetry axis of the torus is aligned with the radio jet. Radiation from the continuum source and BLR can escape along the poles of the torus where it is scattered toward us, giving this radiation a partial linear polarization. The wavelength-independence of the polarization indicates electron scattering as the process responsible for the reflection.

The FOC ultraviolet observations confirm this geometry and locate the electron scattering medium close to and around the nucleus.

The quasar OI 287 has a high and constant optical polarization but unlike most radio sources, is oriented parallel to the radio axis, a quiescent optical flux, 


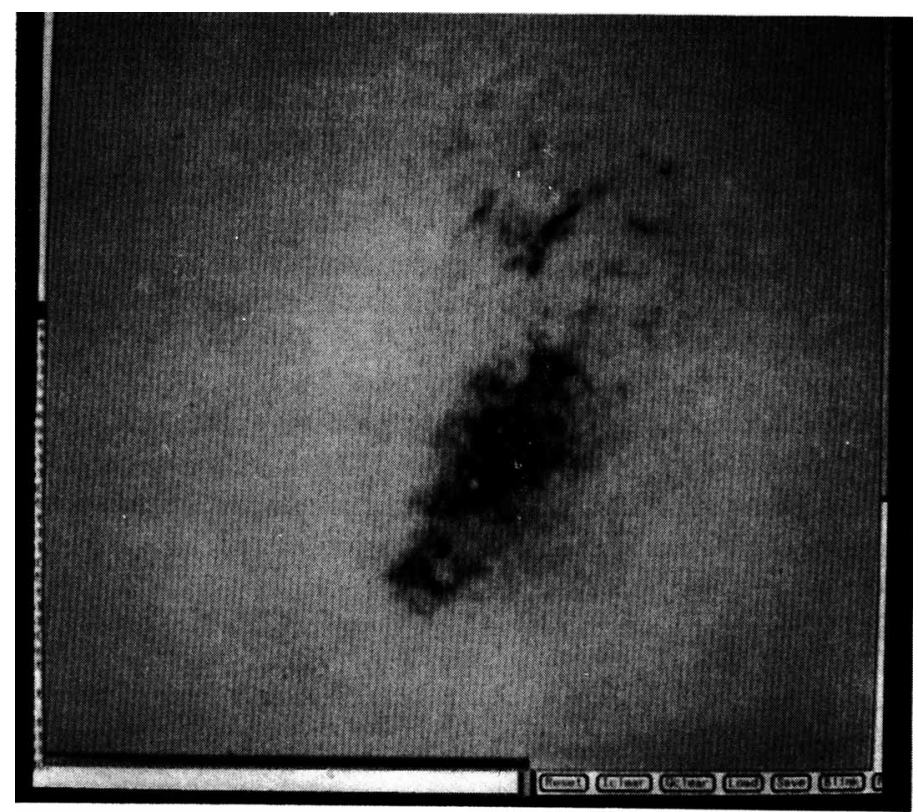

Fig. 10 This FOC image of NGC 1068 taken in [O III] shows the emission-line clouds arranged in a biconical structure. The cloud sizes are of the order of $10 \mathrm{pc}$.

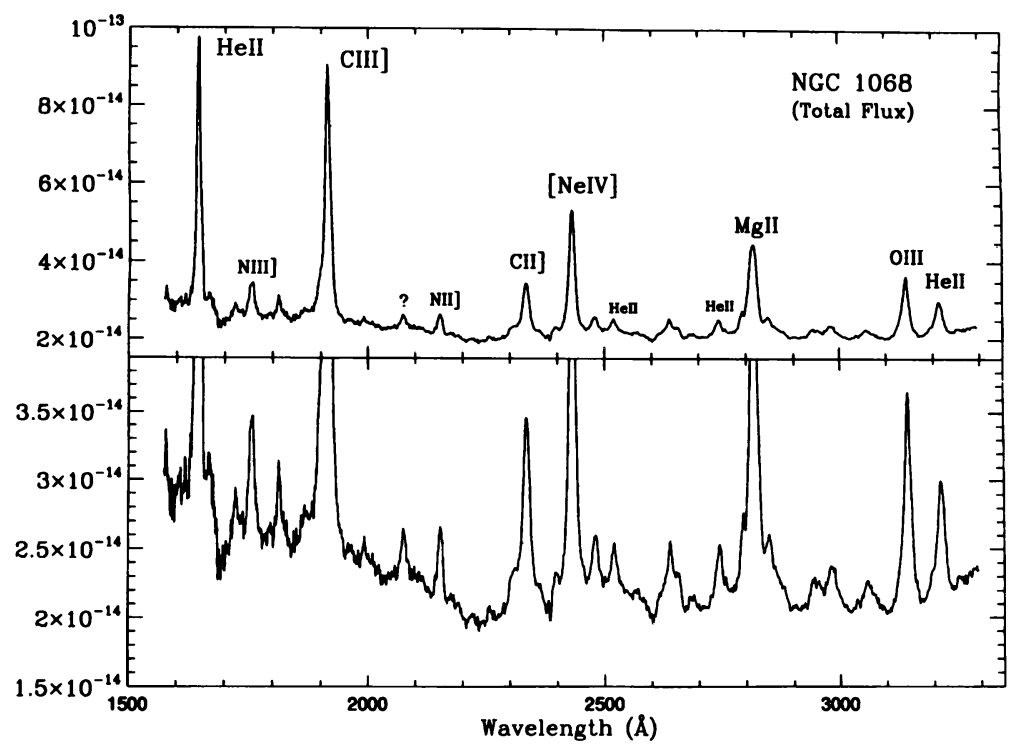

Fig. 11 Total and polarized spectra of NGC 1068. The starlight dilution at the red end of the total spectrum is apparent. 
and a lobe-dominant radio source. It is a possible transition object.

Previous studies have led to the picture of an edge-on thin dusty torus occulting a featureless continuum (FC) source and a broad-line region. The FC source and the BLR are seen only in reflected polarized light. In the unified models, this makes OI 287 a "Quasar 2," analogous to the Type 2 Seyfert galaxies and the narrow line radio galaxies.

Observations of OI 287 were carried out with the FOS $4.3^{\prime \prime}$ square aperture and covered the wavelength range $1200 \AA-2300 \AA$ (Antonucci, Kinney \& Hurt, 1993). The HST spectrum, Fig. 12, shows that OI 287 does have two spectrally resolved and statistically significant absorption features on the blue wing of the C IV line! The absorbers have substantial velocities relative to the quasar $(\sim 7100 \mathrm{~km} / \mathrm{s}$ and $\sim 4000 \mathrm{~km} / \mathrm{s}$ ), and also substantial velocity ranges. These features are likely to represent material outflowing from the quasar. Thus, they are similar to BAL absorbers. The percent polarization is fairly constant with wavelength in OI 287. This wavelength-dependence is inconsistent with an origin due to transmission through dust grains like those in the Milky Way disk and in nearby galaxies, but can easily be accounted for by electron scattering in a region between the BLR and the NLR.

The data for OI 287 shows that it is probably an "edge-on" quasar, with the continuum and BLR sources occulted by an opaque, but geometrically thin dusty disk. By analogy, the UV spectrum of OI 287 also provides support for the notion that $\mathrm{BAL}$ objects may have obscuring disks relatively close to edge-on to our line of sight.

Observations of Mrk 78 were carried out with the FOC at wavelengths including [O III], and at the nearby continuum (Capetti, Macchetto, Sparks \& Boksenberg, 1994).

Two regions of high luminosity within a fainter extended halo are seen in the data, Fig. 13; they are located East and West of the galaxy's center and show smaller scale structures. The halo is more extended towards the West. In this region, it has several bright knots and two bright arcs with an overall biconical shape.

The cone has a full opening angle of $40 \pm 5$ degrees, and its axis is at a position angle of $67 \pm 2$ degrees from the North. The central region of the cone is completely obscured in an area of $0.8^{\prime \prime}$ in size along the E-W direction and displaced from the center of the cone by $0.2^{\prime \prime}$. Its angular size is $0.8^{\prime \prime}$ (corresponding to $900 h_{50}^{-1} \mathrm{pc}$ ) in the direction perpendicular to the cone axis.

The biconical structure observed in the [O III] image, strongly indicates that the origin of the ionization is a central and compact source whose emission is restricted to a well defined solid angle by a parsec size thick accretion torus.

These results define the geometry of this region: obscuring material prevents the UV emission from the central ionizing source to escape along the line of sight; the same thing happens to any [O III] emission from the innermost gas. The continuum (starlight) emission on the other hand comes from an external region, and it is not 


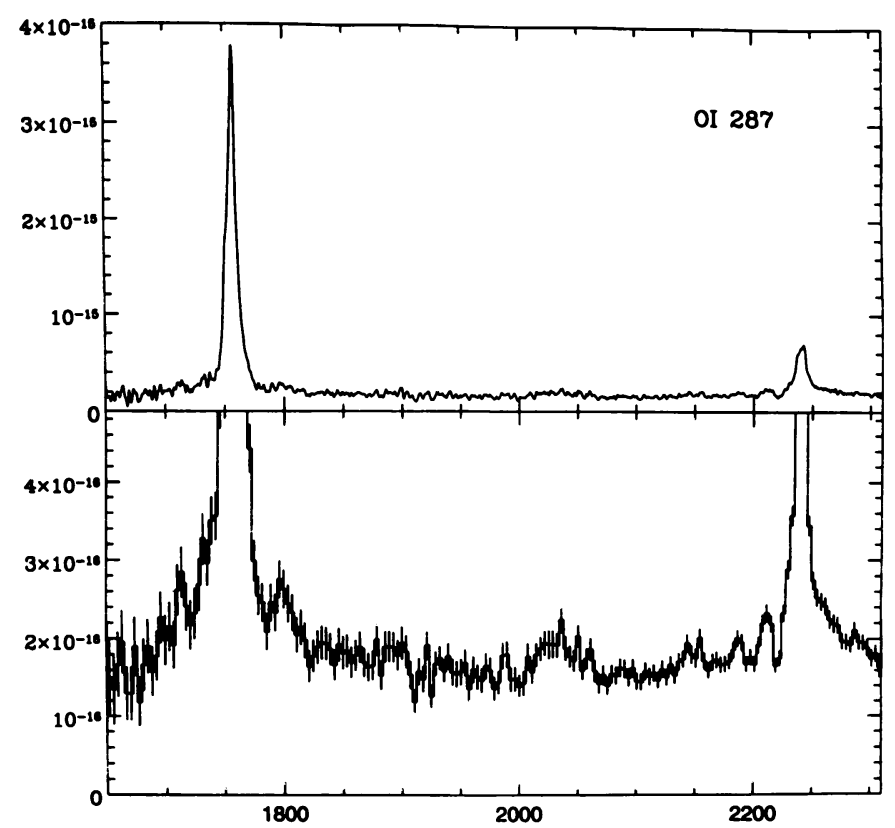

Fig. 12 The spectrum of OI 287 as observed with the FOS. Note the absorption features on the blue wing of C IV.

affected by the nuclear obscuring material.

The large size of the [O III] obscured region, $900 h_{50}^{-1} \mathrm{pc}$, suggests that absorbing material (i.e., dust) is required on a much larger scale in addition to the torus (few parsecs size) obscuring the Seyfert 2 nucleus. The geometry of the obscuring material must be related to, or even determined by, the central torus, since otherwise we would require two different obscuration mechanisms, one for the nuclear ionizing source and the other for the central region.

This result is a strong confirmation of the occultation/reflection model for Seyfert 2 galaxies, with a compact central source as the origin for the ionization. The UV emission from this source is obscured, as confirmed by the total lack of far UV emission in the F130M data.

Dust surrounding the accretion torus is needed to explain the extended obscured central region. The origin for the overall geometric configuration is likely to be connected with the ionizing source and/or with outflowing gas.

The alignment of the extended [O III] emission with the cone of [O III] emission supports the idea of a common origin for the ionization. Nevertheless, the compact 


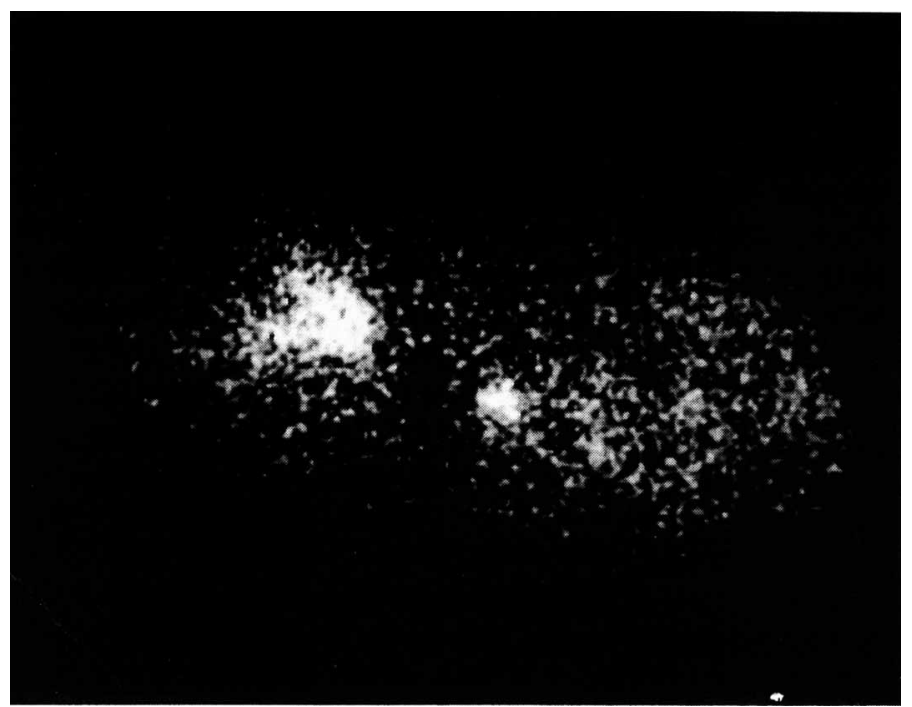

Fig. 13 FOC observations of Mrk 78 in [O III] showing two regions of high luminosity within a fainter extended halo, which has a biconical structure with $40^{\circ}$ opening angle.
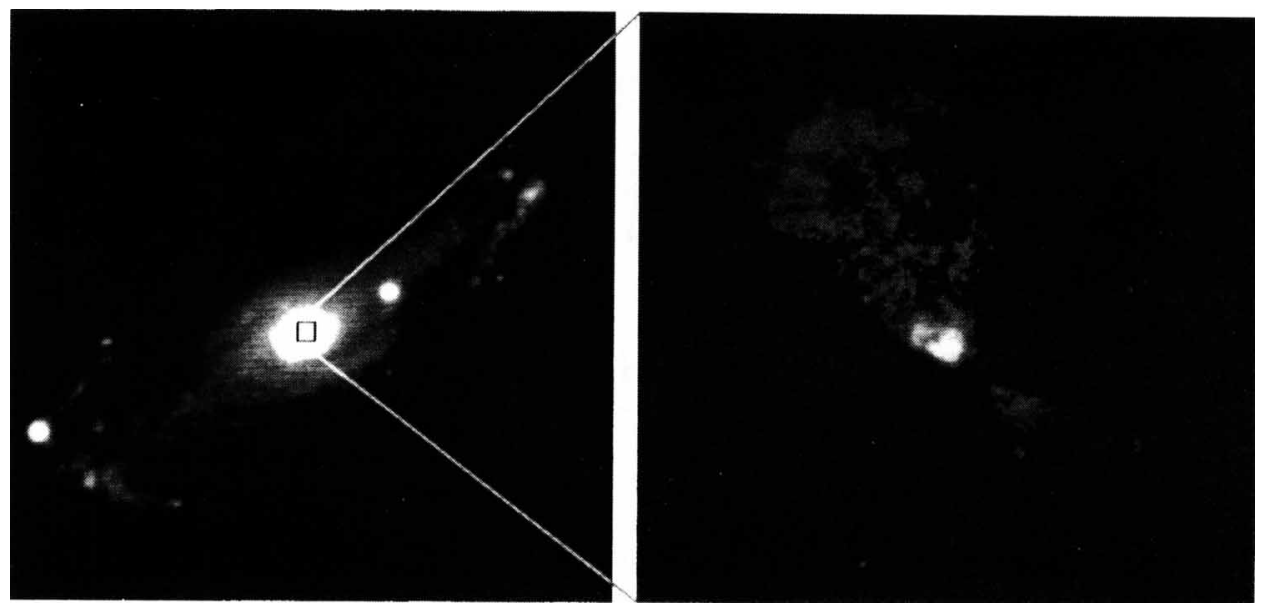

Fig. 14 Ground-based (left) and HST (right) images of NGC 5728. The HST data shows [O III] extended emission $\left(8^{\prime \prime}\right)$ with a well defined biconical structure. The opening angle is $60^{\circ}$. 
and extended emitting gas display different dynamic behaviours.

The radio emission is misaligned with respect to the [O III] emission by about 25 degrees; there is no direct morphological association between the radio and [O III] emission. This strengthens the interpretation of a pure photoionization from a nuclear source as the origin for the overall emission line region.

The observed UV flux is at least three order of magnitude smaller than the flux required to ionize the NRL, corresponding to a column density of $N_{H}>$ $0.5 \times 10^{22} \mathrm{~cm}^{-2}$. For an isotropic source, the corresponding far infrared luminosity would greatly exceed the value obtained from the IRAS observations; thus, the dust must be heated by a highly anisotropic source, as expected in the case of an accretion torus, with reflecting funnel walls.

NGC 5728 is one of the galaxies which are known to exhibit ionization cone structure. Emission-line images (Pogge 1989) reveal a fan-shaped region of high excitation gas extending $\simeq 8^{\prime \prime}$ towards the $S E$ from a bright core.

HST observations were made using the Planetary Camera by Wilson et al. (1993). Exposures with filters were taken in the $\mathrm{H} \alpha+[\mathrm{N} \mathrm{II}] \lambda \lambda 6548,6583$ emission, and in the [O III] $\lambda \lambda 4959,5007$ emission. Exposures were also taken through nearby filters to record the off-band continua.

The dominant continuum feature is an off-centered, bar-shaped region which extends from a peak near the nucleus to another peak $2.2^{\prime \prime}(400 \mathrm{pc})$ to the east. A third peak lies $\simeq 1.5^{\prime \prime}$ to the NW with a prominent dark band separating the two. This dark band coincides with the apex of the cones.

The $\mathrm{H} \alpha+[\mathrm{N} \mathrm{II]}$ and [O III] continuum-subtracted images, Fig. 14, reveal that the high excitation gas near the nucleus of NGC 5728 is contained within two conical regions with a common apex. No [O III] emission is detected at the apex and the $\mathrm{H} \alpha+[\mathrm{N} \mathrm{II}]$ emission is weak there. The cone axes are colinear to within the uncertainties in defining them and the opening angles of the two cones are also very similar at $\simeq 55^{\circ}-65^{\circ}$. Profiles of the $[\mathrm{O}$ III] and $\mathrm{H} \alpha$ brightness plotted perpendicular to the cone axis in the SE cone show flat-topped and double-peaked profiles $0.25^{\prime \prime}-1^{\prime \prime} \mathrm{SE}$ of the line maximum. Flat-topped transverse spatial profiles can result when the illuminated gas lies in a disk, rather than being distributed with spherical symmetry. Double-peaked transverse profiles indicate a hollow cone and are also apparent in the ionization cone of Mrk 78 (Capetti et al. 1994); such profiles may result from "shadowing" of the ionizing radiation by optically thick gas closer to the nucleus along the axis of the cone.

The morphologies of the ionized gas, and the continuum emission can be well understood in the context of the torus model for Seyfert galaxies. The torus is located at the apex of the ionization cones, with the axis of the torus aligning with that of the cones. 


\section{Conclusions}

The observations described in this review constitute far less than a quarter of the data taken with the HST in the field of AGN, and currently being analyzed. It is clearly an impressive array of exciting scientific results, which impose tight new observational constraints on our current physical models. The HST has the unique capability of probing the innermost regions of active galaxies, precisely where the most important physical phenomena are taking place. These observations will undoubtedly contribute to the solution of some of the more difficult problems, such as: what is the real nature of the central energy source; what are morphology and physical properties of the inner accretion tori; what is, and where is the region responsible for the featurless continuum, etc. To solve these and a number of other related problems will require, however, not only the precise observations of which HST is capable, but correlated observations at other wavelength ranges.

\section{References}

Antonucci, R.R.J., and Miller, J.S. 1985, ApJ, 297, 621

Antonucci, R.R.J. 1993, Annu. Rev. Astron \& Astrophys, 31, 473

Antonucci, R.R.J., Kinney, A.L., \& Hurt, T. 1993, $A p J$, in press

Barnes, J. \& Haernquist, L. 1991, ApJ L, 370, L65

Boksenberg, A. 1992, in Science with the Hubble Space Telescope, ed. P. Benvenuti \& E. Schreier, Munich: ESO, 61

Boksenberg, A., Macchetto, F., Albrecht, R., et al 1992, Astron \& Astrophys, 261, 393

Boksenberg, A., Macchetto, F., Catchpole, R., et al 1993a, Astron \& Astrophys, submitted

Boksenberg, A., Macchetto, F., Catchpole, R., et al 1993b, Astron \& Astrophys, submitted

Capetti, A., Macchetto, F., Sparks, W. B., \& Miley, G.K. 1994, Astron $\&$ Astrophys, to be published

Capetti, A., Macchetto, F., Sparks, W.B., \& Boksenberg, A. 1994, ApJ, in press

Code, A.D., et al 1993, $A p J L, 403$, L63

Crane, P., et al 1993, ApJ $L, 402, \mathrm{~L} 37$

Evans, I.N., Ford, H.C., et al 1991, ApJ $L, 369$, L27

Evans, I.N., Tsvetanov, Z., Kriss, G.A., Ford, H.C., Caganoff, S., \& Koratkar, A.P. 1993, ApJ, 417, 82

Fillipenko, A. V., Ho., L.C. \& Sargent, W.L.W. 1993, ApJ L, 410, L75

Ford, H.C., et al 1993, ApJ, submitted

Fraix-Burnet, D., Golombek, D., Macchetto, F., Nieto, J.-L., Perryman, M.A.C. \& Di SeregoAlighieri, S. 1991 AJ, 101, 88

Heckman, T., 1992, ÂP Conference Proceedings, ed Holt S., \& Urry, M; AIP, 595

Hurt, T., Antonucci, R.R.J., \& Miller, J.S. 1993, Proc. 182nd AAS, 789, in preparation.

Jaffe, W., Ford, H.C., Ferrarese, L., van den Bosch, F. \& O'Connell, R. W. 1993, Nature, 364, 213

Lauer, T.R., Faber, S.M., Lynds, C.R., et al 1992, AJ, 103, 703

Macchetto, F. 1991a, Heidelberg Conf. on Active Galactic Nuclei, ed., S.J. Wagner, W.J. Duschl, Springer-Verlag: Berlin, 325

Macchetto, F., 1991b, 7th IAP Conf. in Extragalactic Radio Sources from Beams to Jets, et. Sol. $A, 309$

Macchetto, F. 1992, in Science with the Hubble Space Telescope, ed. P. Benvenuti \& E. Schreier, Munich: ESO, 73

Macchetto, F., et al 1991a, ApJ L, 369, L55

Macchetto, F., et al $1991 \mathrm{~b}, A p J L, 373, \mathrm{~L} 55$ 
Macchetto, F. 1993, Mem. S.A. It., 64, 11

Macchetto, F., Biretta, J.A., \& Sparks, W.B., 1992, Proc 182nd AAS in Comparison of HST and VLA Images of the M87 Jet, Arizona, BAAS, 24, 1183

Mackenty, J.W. 1986, ApJ, 308, 571

Mackenty, J.W., Simkin, S.M., Griffiths, R. E. \& Wilson, A.W. 1993, ApJ, submitted

Miller, J.S. \& Antonucci, R.R.J. 1983, ApJ L, 271, L7

Pogge, R.W. 1989, ApJ, 345, 730

Sanders, D.B., Phinney, E.S., Neugbauer, G., Soifer, B.T., \& Mathews, K. 1989, ApJ, 347, 29

Schweizer, F. 1982, ApJ, 252, 455

Shaya, E.J., Dowling, D.M., Currie, D.G., Faber, S.M., \& Groth, E.J. 1993, ApJ, submitted Sparks, W.B., Biretta, J.A., \& Macchetto, F. 1993, ApJ, submitted

Thomson, R.C., Mackay, C.D. \& Wright, A.E. 1993, Nature, 365, 133

Whitmore, B.C., Schweizer, F., Leitherer, C., Borne, K., \& Robert, C. 1993, AJ, 106, 1354

Wilson, A.S., Breatz, J.A., Heckman, T.M., Krolik, J.H., \& Miley, G.K. 1993, ApJ L, in press 